\title{
La frontière, une ligne impossible
}

\author{
Anne-Christine Habbard \\ Université de Lille III
}

Je m'intéresse ici à la frontière nationale, cet objet politique très étrange, à la fois condition de possibilité de l'État-nation et sa limite. Mon propos, après une rapide généalogie de la frontière nationale, visera à étudier ce caractère aporétique de la frontière; je soutiendrai que si elle apparaît comme périphérique, elle est au contraire au cœur même de la modernité politique, en ce sens qu'elle est décisive dans la construction de ce que l'on appelle l'identité, nationale ou culturelle; le problème est que la frontière apparaît comme un 
lieu (ou plutôt un non-lieu) injustifiable théoriquement. On se trouve donc dans cette position inconfortable d'avoir un élément essentiel d'une définition (de la nation) qui est luimême injustifiable ou indéfinissable. Je reviendrai ensuite sur les conditions de possibilité théoriques de la frontière, et particulièrement l'importance de la géométrie. Cela me mènera à poser la thèse que la frontière est le dernier reliquat de la métaphysique dans un monde post-métaphysique.

\section{L'État-nation et le principe de territorialité}

La frontière nationale est indissociable de la territorialité, forme spécifique de la souveraineté sur laquelle repose l'Étatnation moderne. Auparavant, le souverain régnait sur une tribu, un peuple (le « roi des Francs »); l'allégeance était relationnelle et non territoriale, définie par le lignage, les liens de sang, la caste, la vassalité ou le clan ethniquement défini. Les royaumes eux-mêmes n'étaient pas homogènes, mais une mosaïque d'enclaves et d'ex-claves (Hirst, 2005, p. 31). Ce n'est que depuis les traités de Westphalie au XVIIesiècle que la communauté politique est conçue comme une unité territorialisée, où le gouvernement politique des individus passe par le contrôle exclusif et absolu d'un espace homogène délimité : l'identité est désormais territorialement construite plutôt que culturellement donnée. Cela ne signifie pas que les régimes politiques précédents ne marquaient pas leur territoire, mais jusqu'à l'invention de l'État-nation, le territoire ne constituait jamais un élément à soi seul décisif pour déterminer l'entité politique; il était toujours combiné à un principe de lignage ou d'allégeance à une personne (roi, empereur). De même, les frontières ou démarcations physiques, 
telles la Grande Muraille de Chine ou le Mur d'Hadrien au Royaume-Uni, ne sont pas des frontières au sens moderne du mot, mais davantage des marqueurs symboliques de puissance. Loin de signaler une limite, il s'agit plutôt d'indiquer la projection vers un au-delà, que l'on menace, que l'on hante ou dont on annonce la conquête souhaitée. En ce sens, la frontière pré-nationale indique un au-delà de la frontière; elle n'est pas tant une délimitation entre intérieur et extérieur qu'un signe extérieur de puissance et n'est donc pas indispensable à l'exercice du pouvoir. Avec l'État-nation, en revanche, nous assistons au fait nouveau et inédit que l'identité politique devient liée à une portion délimitée du globe terrestre: le territoire n'appartient qu'à une seule autorité, et non à un autre État, ou à un autre pouvoir, tel l'Église; inversement, chaque sujet n'est soumis qu'à un seul souverain. Là où, au Moyen Âge, Dieu était considéré comme propriétaire de la totalité du globe, cette omnipotence se retrouve, diffractée et indéfiniment répétée, dans chacun des territoires nationaux. Hobbes disait du gouvernement qu'il était un Dieu mortel : l'État a en effet hérité de tous les attributs du Dieu médiéval - omnipotence, éternité, ipséité, causa sui, inconditionnalité, absoluité (Derrida, 2003, p.32-33), dont le dominium spatial. Chaque souverain règne sur sa petite portion du monde tel un Dieu.

Le principe de territorialité a ainsi créé un espace homogène, aboli les hiérarchies et fiefs locaux, et permis une application uniforme de la loi. La transition graduelle d'une entité politique fondée sur le statut à une entité fondée sur l'espace devint ainsi un moyen extraordinairement efficace pour gouverner, en ouvrant la voie à un plus grand contrôle politique, une meilleure collecte et distribution des ressources, une dépersonnalisation des relations et une réification du 
pouvoir. Ainsi, la territorialité est devenue un instrument génial de pouvoir des gouvernements nationaux émergents, tout en fournissant un discours parfaitement adapté à la sensibilité politique émergente de l'individu libéral. C'est également à partir de l'émergence de la nation qu'a été rendue possible la notion d'identité politique, prélude au nationalisme (Hobsbawm, 1990); la politique prit un tournant spatial: l'identité et l'autorité politiques devinrent inséparables du contrôle, de la définition, de la représentation et de la connaissance du pays.

Ce territoire national suppose de tracer des frontières : contrairement à l'empire caractérisé par la distinction entre centre et périphérie, le territoire national n'existe que comme surface plane et uniforme (juridiquement, culturellement, politiquement) délimitée par des lignes-limites, les frontières. Cette spatialisation de l'État entraîne également la représentation ou le mythe de la sédentarité d'une population homogénéisée par l'histoire, la culture ou la langue; c'est-à-dire que l'État est conçu comme un espace isomorphe, délimité et clos. Depuis l'avènement de l'État-nation, la sédentarité, c'est-àdire un rapport premier et durable au sol, est systématiquement prise pour un élément déterminant du politique. D'où l'émergence de toute une rhétorique des racines et de l'enracinement, qui n'ont jamais autant été célébrés que depuis l'avènement de cette forme historiquement contingente de l'État-nation.

La frontière fait donc de l'État un plat Tupperware : nous pouvons l'ouvrir, le fermer, le vider de ses éléments indésirables, le remplir d'éléments désirés. À l'échelle internationale, cela signifie une multiplicité d'États 
indépendants comme autant d'espaces fermés sur eux-mêmes, ou, du moins, capables de l'être. Comme le souligne le politicologue Ernst Gellner, la carte du monde moderne ressemble «à un Modigliani; il y a très peu d'ombragés; les surfaces sont planes et nettement séparées les unes des autres, on voit généralement clairement où l'une commence et l'autre finit, et il n'y a pas d'ambiguïté ni de superposition » (Gellner, 1983, p. 139-140). Les frontières, les lignes de séparation sont minutieuses et bien tracées, les contours sont nets; il n'y a plus de place pour le flou, le fondu, l'indécis, l'entre-deux (Ferguson, Gupta, 1992). En d'autres termes, la séparation en tant que telle ne pose pas de problème, et la discontinuité n'est, elle, pas remise en question. La frontière est donc tenue pour acquise : elle peut être célébrée ou décriée, mais elle est toujours une « évidence ». La justice peut bien faire l'objet de débats au sein de cet espace national, mais la frontière comme délimitation de l'espace ne fait pas, elle, l'objet d'une réflexion de justice. Cela a des conséquences politiques importantes - notamment une pseudo-évidence du droit à la restriction de l'immigration.

\section{Une frontière inexpliquée et inexplicable}

Or cet objet tellement naturel de la modernité politique n'est jamais élucidé de façon satisfaisante par les penseurs de cette modernité. Si la modernité a fait du politique le résultat d'un contrat, d'une convention - le fameux contrat social -, alors on voit bien comment la justice désormais peut faire l'objet d'une délibération rationnelle et collective; elle n'est plus donnée par aucune nature, aucun dieu, ni offerte dans aucune intuition, mais doit être construite et justifiable. La justifiabilité est en ce sens le propre de la modernité politique; mais si on comprend 
bien pourquoi opter en faveur du politique plutôt que de rester à l'état de nature (Hobbes : pour vivre en sécurité; Locke : pour garantir les libertés; Rousseau : pour réaliser ma liberté), la raison de la séparation des individus en communautés multiples reste obscure: pourquoi plusieurs communautés politiques plutôt qu'une seule? Le contrat social repose sur une conception de l'individu libre et rationnel, pas sur une conception de l'individu parlant français, allemand; de l'individu pachtoune, kurde ou québécois: la seule présupposition est que nous sommes des personnes libres, morales, égales. Il n'y a donc aucune raison de penser que notre contrat social doive être limité à quelques-uns, géographiquement proches. En d'autres termes, la frontière nationale, qui existe de fait, bien sûr, et qui est le résultat de processus historiques, culturels et politiques très divers, n'a pour autant, en termes normatifs, aucune légitimité rationnelle indiscutable.

Les arguments en faveur de la partition peuvent se classer en deux grandes catégories : l'une posant l'existence de groupes nationaux ou culturels distincts pré-politiques, existence qui est à la fois une nécessité et un bien rationnels; et l'autre, issue de la théorie démocratique, posant que la liberté politique suppose une communauté limitée, car elle implique que le sujet de la loi en soit aussi l'auteur; l'autonomie ou l'autogouvernement du demos suppose donc que ce dernier soit fini ou limité. Dans un cas, c'est la pré-existence de groupes culturels qui est légitimée; dans l'autre, c'est leur construction.

Supposons d'abord que la frontière résulte de la (dé)limitation du peuple qui y réside : l'argument pose alors que le territoire doit être limité et l'espace, fragmenté, car il faut 
bien autoriser des peuples différents à coexister selon leur propre conception du bien. Plusieurs arguments découlent de cette position. Dans cette perspective d'une séparation naturelle des peuples, l'absence de frontière et une institution cosmopolitique seraient coercitives, ou en tout cas représenteraient une contrainte à l'égard des communautés, notamment celles qui ne sont pas libérales (Rawls, 1993; Chauvier, 1999; Walzer, 1992). Mais il faut ici rappeler que le droit international impose déjà des contraintes normatives sur les communautés non-libérales, de sorte qu'il n'est déjà plus possible de vivre une conception $d u$ bien qui serait radicalement antagoniste avec des principes de respect des droits humains. Il faudrait en outre envisager une clôture quasi hermétique de la communauté, et notamment une interdiction d'émigration, pour qu'une telle communauté puisse préserver sa conception du bien; enfin, une communauté partageant une conception du bien compatible avec le droit international des droits de l'homme est envisageable sans avoir à recourir à une frontière d'exclusion.

Le second argument pose que la communauté limitée est porteuse de sens; elle agit comme vecteur d'identité pour l'individu, en lui fournissant l'aune à laquelle il mesure ses valeurs et ses choix possibles; il est donc essentiel qu'elle puisse se développer séparément. La spécificité de la communauté nationale ou proto-nationale par rapport à d'autres collectivités est qu'elle est comprise comme complète ou sensée, comme on le voit chez Will Kymlicka ou Joseph Raz et Avishai Margalit, c'est-à-dire qu'elle agit comme un filtre qui nous permet de comprendre et donner un sens à nos expériences de vie. La séparation des communautés est donc un bien, car l'individu ne s'accomplit que dans le cocon de l'une d'entre elles. On le voit, il 
$\mathrm{y}$ a là (comme souvent dans les discussions relatives au nationalisme) un naturalisme et une transition implicite du droit vers le fait : que de nombreux individus soient attachés à leur culture ou nation est un fait, sans pour autant signifier que cela soit normativement indispensable pour devenir un soi. Et même en accordant que l'existence d'un groupe culturel ou national soit importante comme filtre ou aune des options d'existence, il n'est nullement nécessaire de supposer une unique culture pour ce faire. Comme le souligne Jeremy Waldron, il est peut-être important d'avoir accès à des cultures : mais n'est nullement par là démontrée la nécessité d'appartenir à une seule culture pour que soit conféré un sens à mes options ou à mes actions. La culture est donc peut-être indispensable pour l'individu, mais non l'intégrité et l'unicité de la culture (Waldron, 1992, p.107). En d'autres termes, nulle indispensabilité de la frontière entre nations ou groupes nationaux sur ce fondement non plus.

De façon générale, la pluralité des cultures et des nations (le «particularisme») est célébrée comme un bien: i) la diversité culturelle serait un bien en soi, au même titre que la biodiversité et ii) l'altérité serait indispensable à l'identité, dans une version collective et politique de la dialectique de la reconnaissance de Hegel, qui culmine avec la version agonistique schmittienne.

L'argument de la diversité culturelle ne tient guère, à moins d'adopter une vision spéciste de l'humanité. Quant à la version collective de la dialectique hégélienne, elle est très problématique. Car autant il est sensé de poser que l'individu en tant que sujet requiert la validation de l'autre subjectivité pour que soit validée sa liberté, autant il est difficile de soutenir 
qu'un groupe requière un autre groupe pour exister en tant que tel. On peut parfaitement soutenir que la validation objective de l'identité collective provient de la reconnaissance des membres du groupe entre eux (Abizadeh, 2005): la nécessité de la séparation n'est pas prouvée rationnellement et repose, là encore, sur un naturalisme subreptice.

La première série d'arguments ne parvient donc pas à poser la nécessité du pluralisme politique sur le fondement d'une nation, c'est-à-dire d'un groupe ayant une identité collective fixe, définie et délimitée pré-politique. Une telle délimitation n'est jamais possible, et la notion de nation (ou de culture sociétale, pour reprendre le terme de Kymlicka) est toujours posée expost, après la formation politique, même imaginée et fantasmée comme la précédant.

Tournons-nous donc vers la seconde série d'arguments, plus cohérente, qui pose, elle, que la séparation est politique et procède du processus démocratique, plutôt que d'être prépolitique et issue d'une limitation «naturelle» des communautés. La frontière serait alors construite par processus démocratique et apparaîtrait alors justifiable au regard de la raison.

La démocratie est comprise comme l'auto-détermination d'un demos, c'est-à-dire l'exercice du pouvoir par un peuple constitué, et donc limité. Contrairement au libéralisme, qui part de l'égalité des personnes morales et qui récuse donc potentiellement dans son principe la notion même de frontière, le républicanisme, entendu comme théorie démocratique, suppose, lui, d'être particularisant et exclusiviste (Cohen 1999; Abizadeh 2008) : c'est aux membres du peuple de déterminer leur sort. Or le problème surgit lorsqu'il faut déterminer les 
membres de ce peuple : comment, selon quels critères et selon quelle procédure, décider qui est membre? Qui est membre légitime, et surtout, qui est autorisé à décider qui est légitime? Quand bien même tous les membres du corps démocratique seraient-ils d'accord pour être membres, cela serait encore insuffisant: car encore faudrait-il que le demos se justifie auprès de ceux qu'il a exclus, puisque la frontière agit comme principe coercitif à l'égard à la fois des membres et des nonmembres. À ce titre, et dans la mesure où l'on définit la démocratie précisément par sa justifiabilité auprès de tous ceux qu'elle affecte, elle a à se justifier également devant les nonmembres, c'est-à-dire ceux qu'elle exclut. Le problème de l'appartenance légitime au corps démocratique (et le problème corrélatif du droit à exclure), c'est-à-dire de la frontière démocratique, est insoluble par une procédure elle-même démocratique (Whelan, 1983). En d'autres termes, il nous faut sortir de la démocratie pour établir le demos sur lequel la démocratie se fonde : soit on pose un peuple pré-existant à la démocratie (la «nation »), mais dans ce cas on retombe dans le cas de figure précédent et son impossibilité; soit on pose par un fiat arbitraire les limites de l'appartenance du corps du demos, c'est-à-dire les frontières.

Or c'est là exactement ce que l'on constate: il existe toujours un point, politique ou territorial, d'inexplicabilité et d'arbitraire, où la frontière ne peut plus se justifier par nulle raison ou par nulle procédure. La démocratie ne peut justifier sa frontière par ses propres moyens. 


\section{Le coup de force originel}

Quel que soit le biais par lequel on l'envisage, la frontière demeure fondamentalement injustifiable et toujours suspendue à un fait empirique, qu'il soit d'ordre historique ou ethnoculturel. Cela se traduit très naturellement par l'impossibilité de justifier le droit au territoire, pendant géographique de la délimitation politique de la frontière. Quelles que soient les théories adoptées - qu'il s'agisse de la version lockéenne selon laquelle le territoire découle du droit de propriété des premiers contractants rassemblés, de la version kantienne selon laquelle le territoire se justifie par la volonté rationnelle de coexister sous un droit public, ou la version nationaliste (ou « ethnogéographique », pour reprendre le terme d'Avery Kolers) selon laquelle chaque groupe disposant d'un rapport historique et décisif à la terre a le droit de maintenir durablement cette ethno-géographie -, toutes les tentatives pour justifier le droit au territoire échouent. Elles butent toutes au moins sur le test de la particularité (comment justifier le droit à ce territoire-ci, à cet endroit et ainsi délimité?). La frontière, entendue au sens politique ou géographique, demeure toujours suspendue à un arbitraire, un fait, voire un fiat originel : la saisie, ou conquête, d'un territoire par un groupe, qui le légitime rétrospectivement par la mise en place de l'État de droit. C'est bien ce que Rousseau décrit dans le Discours sur l'origine de l'inégalité, comme le montre Mikhail Xifaras :

La fondation initiale des États est un acte de pure puissance et non de droit, qui n'a d'autre légitimité que celle qu'il se donne à lui-même, en offrant la garantie de la légalité aux usurpations qui le précèdent, pour le plus grand profit des riches. Les États n'ont pas plus de droit naturel sur leur territoire que les 
individus qui en sont les sujets n'en avaient sur leurs propriétés avant de se soumettre à la loi. (Xifaras, 2003, p. 341)

Walter Benjamin est peut-être plus explicite encore, qui montre dans sa Critique de la violence que l'État de droit repose toujours sur une violence fondatrice, instituante, qui se dissimule après coup dans l'institution du juridique : mais le droit recèle en son for intérieur une violence qui n'est autre que la violence initiale qui institue tout État. Et cette violence fondatrice, dit Benjamin, n'est autre que celle qui pose les frontières : la violence fondatrice de tout État n'est autre que le fiat de sa spatialisation territoriale. Le juriste Richard Ford le note :

Les frontières qui définissent les juridictions territoriales constituent un paradoxe juridique, car elles sont à la fois absolument contraignantes et désespérément arbitraires. En un sens, toutes les limites juridictionnelles sont arbitraires [...]. Et en même temps, une foi aveugle en la nécessité et la légitimité de ces frontières semble être non seulement le fondement de notre gouvernement, mais la condition de possibilité de tout gouvernement [...]. La logique du gouvernement est la logique de la juridiction : mettez-la en cause et tout ce qui apparaissait solide comme du roc fond comme neige au soleil. (1999, p. 850-851)

La première forme de l'aporie de la frontière émerge ici : rationnellement, il n'existe aucune justification satisfaisante de la séparation des entités politiques : c'est la légitimité même de l'existence des frontières qui est problématique. En d'autres termes, il existe une antinomie entre la dénaturalisation progressive des relations politiques - qui se détachent progressivement de toute assignation naturelle (communauté ethnique, raciale, familiale, etc.) pour être comprises comme des relations de droit et de liberté -, et la frontière, dernier refuge de la naturalité face à une conception normative du 
politique. Plus encore, le fait de penser la structure politique comme circonscrite spatialement, c'est-à-dire fondée sur une distinction cruciale entre "intérieur » et " extérieur », mène à de difficiles paradoxes, dont je crois qu'ils proviennent précisément de cet « angle mort» de la frontière. Ces paradoxes - d'ailleurs plutôt des contradictions - sont bien connus : la contradiction entre intérieur et extérieur, entre une souveraineté domestique illimitée et une souveraineté limitée sur le plan international; entre l'obligation internationale pour l'État d'être une simple «courroie de transmission" entre l'individu sujet de droits et la communauté internationale garante du respect de ceux-ci, et sa présence substantielle, « opaque » et autonome, dans les relations internationales, en tant que personne morale. Ces contradictions, au cœur de «l'énigmatique institution » (Ricœur, 2001, p. 10) qu'est l'État sont, on le voit, finalement toujours liées au territoire et à la frontière.

Mais cette frontière, pour arbitraire et injustifiable qu'elle soit, est pourtant au cœur du sens de la nation : la frontière est géographiquement périphérique, mais politiquement centrale; c'est par rapport à l'extérieur et à l'étranger que se définissent le sens de la nation et l'identité politique (si tant est que ce terme d'identité ait un sens). Celle-ci n'est instituée que par la délimitation de la frontière, au point que «maintenir une frontière permet d'actualiser en permanence la fiction de l'identité nationale » (Le Blanc, 2010, p. 138). Rien de tel que la désignation de l'étranger, ou l'appel à la défense du territoire, pour mobiliser les puissants venins du nationalisme: l'«intégrité du territoire» devient une fin en soi et la contradiction avec le droit à l'auto-détermination des peuples, souvent relevée dans le droit international, n'est jamais telle 
que l'on mette en question les frontières, supposées être des lignes absolues, quoique parfois disputées. Le droit international est intrinsèquement conservateur (Shaw, 1997; Sidgwick, 2005 [1898]; Kolers, 2009) et accorde le bénéfice du doute à l'État existant, quelle que soit sa légitimité démocratique. Carl Schmitt est en ce sens typique lorsqu'il définit l'essence du politique comme étant la distinction entre ami et ennemi : sans frontière, cela serait tout simplement impossible, et il nous faudrait alors re-définir l'identité politique tout entière.

Qu'est-ce qui explique ce rôle indispensable et pourtant impensable de la frontière, cette ligne imaginaire? Comment cette ligne qui, comme toute ligne mathématique, est un trait à la limite de l'inexistence, un simple trait sur une carte, comment donc cette ligne évanescente en est-elle venue à jouer un rôle aussi décisif de notre auto-définition ou identité, nationale, sociale et même morale?

\section{La frontière, vestige de la métaphysique}

Il faut y voir, je pense, des raisons métaphysiques, liées à la construction de l'État moderne et à la conception de l'espace qui le sous-tend. La frontière n'a en effet pris ce pouvoir exorbitant qu'au prix d'un subterfuge naturaliste qui fait du territoire de l'État le dernier vestige de la métaphysique, à une ère post-métaphysique.

En effet, l'État moderne est un héritier des attributs métaphysiques de l'individu. La modernité conceptualise en effet l'État, entité libre en tant qu'héritière de la liberté de ses membres, sur le modèle de la personne, dont il garde les 
qualités essentielles: souveraineté, liberté, indépendance, capacité d'action. On connaît les conséquences de cet acte philosophique en droit international : la scène internationale est une pluralité de personnes libres et égales, et l'équivalent des droits de la personne devient les "droits fondamentaux » des États, dont les trois principaux sont le droit de conservation (droit à la vie et à l'existence), le droit d'indépendance (c'est-àdire de "vie privée», ce qui implique le principe de nonintervention dans les affaires intérieures) et le droit à l'égalité. Un véritable isomorphisme entre individu et État s'est créé. La liberté dans la modernité sera toujours entendue comme i) présupposant l'existence d'un corps (propriété de soi) et ii) nécessitant une extériorisation dans le monde (Descartes "comme maître et possesseur de la nature », Locke, Kant, Hegel). C'est le rôle, pour l'État, du territoire, qui correspond à la propriété de soi de l'individu, ce corps dont il ne peut être détaché et qui devient, dans la théorie de l'État, le territoire sans lequel il ne peut exister. Le territoire substantialise et ontologise l'État; il lui donne chair et corps. Mais il lui donne aussi l'espace extérieur réel sans lequel aucune liberté n’a de sens.

Tout se passe comme si la distinction entre souveraineté de l'État et propriété ne posait aucun problème, sauf dans le cas du sol. Est maintenue à tout prix la possibilité pour l'État de posséder son sol. Le rapport de l'État à son territoire passe par un moment domanial, inévitablement. D'autres illustrations pourraient ici être évoquées, telle la guerre civile ou le droit des immigrés, que nous ne pouvons développer ici mais qui soulignent tout autant ce moment nécessairement propriétariste de l'État (Xifaras, 2004). Celui-ci ne peut rester en permanence État des propriétaires, c'est-à-dire gardien des 
propriétés privées, qu'il est pourtant censé être. En tant que limité territorialement, il est contraint, à des moments décisifs de l'exercice de sa souveraineté, de devenir propriétaire privé.

La distinction entre souveraineté territoriale et propriété privée est notoirement difficile à établir; et toutes deux sont également difficiles à justifier : elles reposent toutes deux sur un moment "magique », auquel nous décidons de croire et qui crée l'attachement ou engendrerait le droit. Nous assistons à un lent processus de démythification, de substantialisation et de désenchantement de l'État-nation, mais sa substantialité même demeure « un facteur de retardement du désenchantement [...]. L'État-nation a pris la relève des anciens systèmes métaphysiques. » (Cheneval, 2005, p. 257)

Or la frontière comme dernier avatar de la métaphysique est rendue possible par la nouvelle conception de l'espace qui émerge à la modernité : on la voit affleurer dès le début du $\mathrm{XV}$ esiècle avec l'invention de la perspective en art et le développement de la cartographie, mais elle sera pleinement théorisée avec Descartes. Cette nouvelle conception de l'espace en fait une res extensa plane, isotropique, vide, infinie ou indéfinie, et conçue comme infiniment divisible (Descartes, 2000, [1651]; Jammer, 1993, p. 53 sq.); l'espace cartésien est l'espace de la multiplicité et de l'exclusion, celui-là même qui rend possible le système international des États, souverains, multiples et exclusifs. C'est seulement sur le fondement de cette conception de l'espace que va être rendu possible le territoire comme « contenant», remplissable et vidable à merci. 


\section{Le rôle de la cartographie}

Cette conception de l'espace rend possible la frontière par la médiation de la cartographie : en effet, l'une des spécificités de cette spatialité moderne est qu'elle est indissociable de sa représentation; régner sur un espace signifie contrôler aussi les représentations de cet espace. Gouverner un pays nécessite une connaissance de ce territoire; considérer ce pays comme une unité dirigée par un dirigeant suppose aussi de le voir comme un. En d'autres termes, l'État-nation ne peut exister sans une représentation de son espace, c'est-à-dire sans une carte. Pour que l'espace devienne un territoire homogène et uniforme, il doit être représenté comme tel, notamment grâce à la représentation de sa limite, c'est-à-dire des frontières (Biggs, 1999, p. 385). Les cartes de Cassini en France au XVIII siècle sont révélatrices: le pouvoir gouvernemental s'est accru et renforcé au fur et à mesure de la publication de ces cartes de France.

La spécificité de la modernité est donc que la représentation précède la réalité territoriale. Jean Baudrillard note, dans une phrase restée célèbre, que «la carte précède le territoire», elle l'engendre et le rend possible (Baudrillard, 1985, p. 10). La cartographie a ainsi incarné et rendu possible la vue synoptique de l'État nouvellement formé, tout comme son gouvernement rationnel, impersonnel et uniforme : le regard omnidirectionnel et omniscient du Souverain est indissociable de celui du cartographe. Le tournant scientifique de la modernité a mené à la rationalisation géométrique de l'espace et à l'invention de la cartographie telle que nous la connaissons, dont la conséquence politique fut l'État-nation territorial. La cartographie produit du savoir, mais elle produit tout autant des 
réalités politiques et sociales. Cette capacité d'anticipation et de domination est singulièrement manifeste avec la colonisation : de nombreux travaux montrent, par exemple, comment « l'invention de l'Amérique » (O'Gorman, 1961) est indissociable de cette nouvelle cartographie qui, en représentant les terres nouvellement «découvertes » comme vides, les rendait aussi ouvertes à la conquête et la violence de la colonisation (Nobles, 1993). Le cartographe est oublieux : tout à sa joie de la page blanche, véritable tabula rasa, il oublie les spatialités et les histoires de ceux qui l'ont précédé. La cartographie recrée en ce sens le geste divin du regard créateur ex nihilo. Elle prétend être descriptive alors qu'elle est politiquement et socialement prescriptive.

\section{La ligne moderne}

Si les cartes sont la clef de la constitution de l'État moderne, si l'épistémologie devient un préalable à la philosophie politique, c'est parce que la ligne a désormais changé de sens. En effet, il n'existe certainement aucun geste cartographique plus significatif que la ligne. Les lignes sur une carte sont remarquablement puissantes: d'un seul trait de crayon, à Vienne en 1885, par Sykes et Picot en 1916, à Yalta en 1945, par le juriste Radcliffe à New Delhi en 1947, mais en tant d'autres endroits aussi, des souverainetés sont déterminées, des populations sont divisées, des nationalités sont déterminées, des réalités politiques sont créées, des normes juridiques sont établies. Les lignes sont les premières coupables dans cette territorialisation de l'État. Mais ces lignes ne peuvent bien sûr exister que sur le papier ou, du moins, elles doivent d'abord exister sur le papier pour être ensuite matérialisées. C'est donc 
par la cartographie que la ligne a pris ce sens prohibitif. Certes, la ligne existait, bien sûr, dans les cartographies antérieures et pré-modernes, mais elle indiquait un itinéraire, mettait l'accent sur le lien entre les groupes ou encore indiquait des trajets, des lignes d'affinité. Tout au contraire, les lignes modernes sont des lignes de division, de séparation, de conquête; comme le note Henri Lefebvre, l'espace abstrait et ses lignes renvoient davantage à des prohibitions et des interdictions qu'à des ouvertures ou des sollicitations (Lefebvre, 2000; Ingold, 2007). La ligne est devenue menaçante : « Ne pas déranger! » « Do not trespass! » «Attention, danger! », une ligne droite que l'immigré clandestin doit traverser à ses risques et périls, mais que l'on ne peut plus suivre. Carl Schmitt, dans son Nomos de la Terre, a caractérisé la modernité jusqu'au début du XXe siècle comme dominée par la « pensée linéaire globale » (2003 [1987], p. 87). La modernité inaugure un nouveau sens de la ligne, comme on le voit de façon spectaculaire avec le Traité de Tordesillas en 1494, qui divise le monde en deux pour le partager entre l'Espagne et le Portugal. La ligne tracée sur la carte a rendu possible la frontière, à la fois au sens territorial, mais aussi au sens politique et culturel : l'illusion de la nation, c'est-à-dire de la clôture possible du groupe, d'une délimitation claire et finie à la fois des membres du groupe et des critères d'appartenance au groupe, naît de ce tracé fin et précis sur la carte. Car en vérité les groupes culturels ou nationaux sont impossibles à définir : quels que soient les critères choisis (langue, ethnie, culture, histoire, institution, etc.), ceux-ci ne parviennent jamais à cerner et à définir le groupe en question; parallèlement, il est tout aussi impossible de déterminer avec précision les individus susceptibles d'appartenir au groupe en question; que faire des individus bi-nationaux, que faire des individus qui exhibent 
certains critères mais pas tous? Les groupes se fondent l'un dans l'autre, les frontières culturelles et nationales n'existent que de façon mythifiée : c'est le mythe de la pureté, qui est aussi la pureté géométrique. Un exemple frappant est celui de l'Afrique du Sud durant l'Apartheid: mues par une volonté de pureté ethno-raciale, les autorités définirent différents groupes (Whites, Coloureds, Natives, etc.). L'application de ces critères se révéla (sans grande surprise) impossible et donna lieu à des situations kafkaesques; des membres d'une même famille étaient catégorisés dans des groupes différents; un même individu pouvait se retrouver dans des catégories différentes à différentes époques de sa vie; ne sachant comment désigner les personnes d'origine chinoise, perçues comme racialement supérieures aux "non-Européens», les autorités les désignèrent comme «Blancs Honoraires » (Honorary Whites) (Beinart, Dubow, 1995; Marx, 1986; Jung Park, 2008).

Ce qui est surprenant, ce n'est pas tant l'impossible application de la catégorisation que la catégorisation ellemême, née de l'illusion d'une limite claire entre les groupes nationaux et/ou culturels. Cette illusion de la limite claire provient de notre habitude de la ligne cartographique.

Si donc la modernité politique est marquée par les frontières, dans la mesure où elles sont les marqueurs de notre citoyenneté et de notre pouvoir d'action, alors la ligne sur le papier anticipe, précède et rend possible la modernité.

Paul Klee, dans ses carnets, se demande s'il n'est pas temps de laisser la ligne prendre la fuite, «take a walk» (1961, p. 105). Laisser cette ligne droite de la modernité, surdéterminée, hyper 
accentuée et prohibitive, reprendre du large, la laisser recommencer à signifier des chemins et des cheminements plutôt que des démarcations et des interdictions: vaste programme intellectuel, esthétique, mais aussi, et surtout, moral et politique, car il s'agit de rien de moins que d'apprendre à re-définir nos identités morale, sociale et politique. 


\section{Bibliographie}

ABIZADEH, Arash. (2005), « Does Collective Identity Presuppose an Other? On the Alleged Incoherence of Global Solidarity », The American Political Science Review, vol. 99, n 1, p. 45-60.

—. (2008), « Democratic Theory and Border Coercion: No Right to Unilaterally Control your Own Borders », Political Theory, vol. 36, no 1, p. 37-65.

BAUDRILLARD, Jean. (1985), Simulacres et simulations, Paris, Galilée.

BEINART, William et Saul DuBow. (1995), Segregation and Apartheid in Twentieth Century South Africa, Londres, Routledge.

Benjamin, Walter. (2012 [1929]), Critique de la violence, trad. Nicole Casanova, Paris, Payot.

BIGGS, Michael. (1999), "Putting the State on the Map: Cartography, Territory, and European State », Comparative Studies in Society and History, vol. 41, nº 2, p. 374-405.

CHAUVIER, Stéphane. (1999), Justice internationale et solidarité, Paris, J. Chambon.

Cheneval, Francois. (2005), La Cité des peuples - Mémoires de cosmopolitisme, Paris, Cerf.

CoHEN, Jean. (1999), « Changing Paradigms of Citizenship and the Exclusiveness of the Demos », International Sociology 14, no 3 , p. 245-268.

DERridA, Jacques. (2003), « The "World" of the Enlightenment to Come (Exception, Calculation, Sovereignty)», Research in Phenomenology, no 33, p. 9-52. 
DesCartes, René. (2000 [1651]), Principes de la philosophie, Paris, Paléo.

FORD, Richard. (1999), «Law's Territory (A History of Jurisdiction) », Michigan Law Review, vol. 97, no 4, p. 843-930.

GELLNER, Ernst. (1983), Nations and Nationalisms, Londres, Blackwell.

GuPTA, Akhil et James Ferguson. (1992), «Beyond "Culture": Space, Identity and the Politics of Difference», Cultural Anthropology, vol. 7, no 1, p. 6-23.

HIRST, Paul. (2005), Space and Power - Politics, War and Architecture, Cambridge, Polity Press.

HobBEs, Thomas. (2010 [1660]), Leviathan, New Haven, Yale University Press.

HobSBAWM, Eric. (1990), Nations and Nationalism since 1780, Cambridge, Cambridge University Press.

InGOLD, Tim. (2007), Lines - A Brief History, Londres, Routledge. JAMmer, Max. (1993), Concepts of Space - The History of the Theories of Space in Physics, Mineola, Dover Science Books.

JUng PARK, Yoon. (2008), «Honorary White, or Non-White: Apartheid Era Constructions of Chinese », Afro-Hispanic Review, vol. 27, no 1, p. 123-138.

KYMLICKA, Will. (2001), La Citoyenneté multiculturelle, Paris, La Découverte.

KLEE, Paul. (1961), Notebooks, vol. 1: The Thinking Eye, Londres, Lund Humphries. 
Kolers, Avery. (2009), Land, Conflict and Justice, Cambridge, Cambridge University Press.

LE BLANC, Guillaume. (2010), Dedans, dehors - La condition d'étranger, Paris, Seuil, coll. « La Couleur des idées ».

Lefebvre, Henri. (2000), La Production de l'espace, Paris, Anthropos.

MARGALIT, Avishai et Joseph RAZ. (1990), "National SelfDetermination », Journal of Philosophy, vol. 87, no 9, p. 439-461.

MARX, A. (1996), "Race-Making and the Nation-State », World Politics, vol. 48, no 2, p. 180-208.

NoBLES, Gregory. (1993), « Straight Lines and Stability: Mapping the Political Order of the Anglo-American Frontier», The Journal of American History, vol. 80, nº 1, p. 9-35.

O'GoRMAN, Edmundo. (1961 [1948]), The Invention of America: An Inquiry into the Historical Nature of the New World and the Meaning of Its History, Bloomington, Indiana University Press.

RAWLS, John. (1993), The Law of Peoples, Critical Inquiry, no 20.

RIC@Eur, Paul. (2001), Histoire et vérité, Paris, Seuil.

SchmitT, Carl. (2003 [1950]), The Nomos of the Earth in the International Law of the Jus Publicum Europaeum, trad. G. L. Ulmen, New York, Telos Press.

SHAw, Malcolm. (1997), International Law, Cambridge, Cambridge University Press.

SiDGWICK, Henry. (2005 [1898]), Elements of Politics, New York, Cosmo Classics. 
WALDRON, Jeremy. (1992), «Minority Cultures and the Cosmopolitan Alternative », Michigan Journal of Law Reform, no 25 , p. 751-793.

WALzer, Michael. (1992), "The New Tribalism: Notes on a Difficult Problem », Dissent, no 39, p. 164-171.

WHELAN, Frederick. (1983), « Prologue: Democratic Theory and the Boundary Problem », Nomos 25: Liberal Democracy, New York, New York University Press, p. 13-46.

XIFARAS, Mikhaïl. (2003), «La Destination politique de la propriété chez Jean-Jacques Rousseau», Les Études philosophiques, n으 66, p. 341.

—. (2004), La Propriété. Étude de philosophie du droit, Paris, PUF. 


\section{Résumé}

Je vais m'intéresser à la frontière nationale et à ses conditions de possibilité graphiques, politiques, géométriques et métaphysiques. Ma thèse est que, du fait de la territorialité de l'État-nation, la frontière nationale, loin d'être périphérique, est au contraire au cœur même du politique et au centre de la modernité philosophique en même temps qu'elle en a entravé la pleine réalisation. Or la frontière n'est ni évidente, ni rationnellement légitime, alors même que notre identité politique et sociale n'existe qu'en rapport avec elle. Pour expliquer cette émergence de la frontière comme cœur du politique dans la modernité, je reviendrai sur deux de ses conditions de possibilité : l'une, métaphysique, porte sur la frontière comme ultime vestige de la métaphysique classique, par le biais de la naturalisation de la différence; l'autre, géométrique et graphique, portera sur le sens nouveau de la ligne.

\section{Abstract}

My aim is to investigate the national border and its metaphysical, political, geometric and graphic conditions of possibility. With the advent of the nation-state and its territoriality, national boundaries, far from being on the periphery, are on the contrary at the heart of the polity and of philosophical modernity, while preventing the fulfilment of that very modernity. Now the boundary is neither self-evident nor rationally legitimate, even though we unreflectively use it to define our political and social identities. In order to account for this emergence of the national boundary as a key to political modernity, I will take a look at its metaphysical condition of possibility (the boundary as last remnant of metaphysics in a post-metaphysical age), as well as at its graphic and geometric conditions, focusing in particular on the new role of the line. 